Bull. Korean Math. Soc. 52 (2015), No. 4, pp. 1069-1076

http://dx.doi.org/10.4134/BKMS.2015.52.4.1069

\title{
PERMANENCE OF A TWO SPECIES DELAYED COMPETITIVE MODEL WITH STAGE STRUCTURE AND HARVESTING
}

\author{
Changuin Xu and Yusen Zu
}

\begin{abstract}
In this paper, a two species competitive model with stage structure and harvesting is investigated. By using the differential inequality theory, some new sufficient conditions which ensure the permanence of the system are established. Our result supplements the main results of Song and Chen [Global asymptotic stability of a two species competitive system with stage structure and harvesting, Commun. Nonlinear Sci. Numer. Simul. 19 (2001), 81-87].
\end{abstract}

\section{Introduction}

The dynamic relationship between predators and their preys has long been and will continue to be one of the dominant themes in both ecology and mathematical ecology due to its universal existence and importance [2]. Dynamic behavior of predator-prey models has been studied by many authors. It is well known that permanence is an important topic in predator-prey models. Moreover, in many applications, the nature of permanence is of great interest. Recently, Fan and $\mathrm{Li}$ [12] investigated permanence of a delayed ratio-dependent predator-prey model with a Holling type functional response. Chen [5] studied theoretically on the permanence of a discrete $n$-species foodchain system with delay. Chen [4] analyzed the permanence and global attractivity of Lotka-Volterra competition system with feedback control. Zhao and Teng et al. [26] addressed the permanence criteria for a delayed discrete nonautonomous-species Kolmogorov system. Jiang [29] focused on the permanence and extinction for nonantonomous Lotka-Volterra system. For more

\footnotetext{
Received July 29, 2013; Revised February 4, 2015.

2010 Mathematics Subject Classification. Primary 34K20, 34C25, 92D25.

Key words and phrases. competitive model, permanence, harvesting, stage structure,

This work is supported by National Natural Science Foundation of China (No.11261010 and No.11101126), Soft Science and Technology Program of Guizhou Province (No.2011LKC2030), Natural Science and Technology Foundation of Guizhou Province (J[2015]2025), Governor Foundation of Guizhou Province ([2012]53) and Doctoral Foundation of Guizhou University of Finance and Economics (2010).
} delay. 
research on the permanence behavior of predator-prey models, one can see $[8,11,13,14,15,17,18,23]$. Here we must point out that most of literatures on these predator-prey models are only connected with parameters which are independent of time delay, while in most applications of delay predator-prey models in population dynamics, the need of incorporation of a time delay is often the result of existence of some stage structure $[1,2,9]$. Indeed, every population goes through some distinct life stages [16, 24]. Since the stage survival rate is often a function of time delay, it is easy to conceive that these models will inevitably involve some delay-dependent parameters. Recently, the research work on the permanence of predator-prey systems with delay-dependent parameters is scare. One can see [3, 6, 7, 21, 27].

In 2001, Song and Chen [25] investigated the global asymptotic stability of the following two species competitive system with stage structure and harvesting

$$
\left\{\begin{array}{l}
\frac{d x_{1}(t)}{d t}=\alpha x_{2}(t)-\gamma x_{1}(t)-\alpha e^{-\gamma \tau} x_{2}(t-\tau) \\
\frac{d x_{2}(t)}{d t}=\alpha e^{-\gamma \tau} x_{2}(t-\tau)-\beta x_{2}^{2}(t)-a_{1} x_{2}(t) y(t)-E x_{2}(t), \\
\frac{d y(t)}{d t}=y(t)\left(r_{1}-a_{2} x_{2}(t)-b y(t)\right)
\end{array}\right.
$$

where $x_{1}(t)$ and $x_{2}(t)$ denote the immature and mature population densities of the species one, respectively, to model stage structured population growth, and $y(t)$ represents the density of the species two. The term $\alpha e^{-\gamma \tau} x_{2}(t-\tau)$ represents the immature who was born at time $t-\tau$ (i.e., $\alpha x_{2}(t-\tau)$ ) and survives at the time $t$ (with the immature death rate $\gamma$ ) and therefore represents the transformation from immature to mature. The model is obtained under the assumptions as follows:

(A1) The species one: the birth rate into the immature population is proportional to the existing mature population with a proportionality constant $\alpha>0$; the death rate of the immature population is proportional to the existing immature population with a proportionality constant $\gamma>0$; the death rate of mature population is of a logistic nature, i.e., it is proportional to the square of the population with a proportionality constant $\beta>0$. Only the mature population has competitive ability, and competitive coefficient $a_{1}>0$. E is the harvesting effort.

(A2) The species two: the growth rate of the species is Lotka-Volterra nature, $r_{1}>0, a_{2}>0, b>0$.

(A3) $x_{i}(0)>0, x_{i}(t) \geq 0(i=1,2)$ on $-\tau \leq t \leq 0, y(0)>0$

We know that any biological or environment parameters are naturally subject to fluctuation in time [28]. In 1977, Cushing [10] pointed out that it is necessary and important to consider models with periodic ecological parameters or perturbations which might be quite naturally exposed (for example, those due to season effects of weather, food supply, mating habits, hunting or harvesting seasons, etc.). Thus the assumption of periodicity of the parameters 
is a way of incorporating the periodicity of the environment. In addition, we consider that the sum of prey species born at time $t-\tau$ is $\alpha(t-\tau) x_{2}(t-\tau)$, the sum of prey species that still alive at time $t$ is $\alpha(t-\tau) e^{-\gamma(t) \tau} x_{2}(t-\tau)$. Based on the discussion above, system (1) can be modified as follows

(2)

$$
\left\{\begin{array}{l}
\frac{d x_{1}(t)}{d t}=\alpha(t) x_{2}(t)-\gamma x_{1}(t)-\alpha(t-\tau) e^{-\gamma \tau} x_{2}(t-\tau) \\
\frac{d x_{2}(t)}{d t}=\alpha(t-\tau) e^{-\gamma \tau} x_{2}(t-\tau)-\beta(t) x_{2}^{2}(t)-a_{1}(t) x_{2}(t) y(t)-E(t) x_{2}(t) \\
\frac{d y(t)}{d t}=y(t)\left(r_{1}(t)-a_{2}(t) x_{2}(t)-b(t) y(t)\right)
\end{array}\right.
$$

The initial conditions for system (2) take the form of

$$
x_{i}(\theta)=\phi_{i}(\theta) \geq 0, y(\theta)=\varphi(\theta) \geq 0, \phi_{i}(0)>0, \varphi(0)>0, i=1,2, \theta \in[-\tau, 0],
$$

where $\left(\phi_{1}(\theta), \phi_{2}(\theta), \varphi(\theta)\right) \in C\left([-\tau, 0], R_{+0}^{3}\right)$. For continuity of initial conditions, we require

$$
x_{1}(0)=\int_{-\tau}^{0} \alpha(s) \phi_{2}(s) e^{\gamma \tau} d s .
$$

The principal object of this article is to explore the dynamics of system (2) with the initial conditions (3). We apply the differential inequality theory to study the permanence of system (2) with the initial conditions (3).

The remainder of the paper is organized as follows: in Section 2, basic definitions and lemmas are given and some sufficient conditions for the permanence of the two species delayed competitive model with stage structure and harvesting in consideration are established. In Section 3, we give an example which shows the feasibility of the main results. Conclusions are presented in Section 4.

\section{Permanence}

For convenience in the following discussion, we always use the notations:

$$
f^{l}=\inf _{t \in R} f(t), f^{u}=\sup _{t \in R} f(t),
$$

where $f(t)$ is a continuous function. In order to obtain the main result of this paper, we shall first state the definition of permanence and several lemmas which will be useful in the proving the main result.

Definition 2.1 ([20]). We say that system (2) is permanent if there are positive constants $M$ and $m$ such that for each positive solution $\left(x_{1}(t), x_{2}(t), y(t)\right)$ of system (2) satisfies

$$
\begin{gathered}
m \leq \lim _{t \rightarrow+\infty} \inf x_{i}(t) \leq \lim _{t \rightarrow+\infty} \sup x_{i}(t) \leq M, i=1,2, \\
m \leq \lim _{t \rightarrow+\infty} \inf y(t) \leq \lim _{t \rightarrow+\infty} \sup y(t) \leq M
\end{gathered}
$$


Lemma 2.1 ([22]). If $a>0, b>0$ and $\dot{x} \geq x(b-a x)$, when $t \geq 0$ and $x(0)>0$, we have

$$
\lim _{t \rightarrow+\infty} \inf x(t) \geq \frac{b}{a} .
$$

If $a>0, b>0$ and $\dot{x} \leq x(b-a x)$, when $t \geq 0$ and $x(0)>0$, we have

$$
\lim _{t \rightarrow+\infty} \sup x(t) \leq \frac{b}{a} \text {. }
$$

Lemma 2.2 ([19]). Consider the following equation:

$$
\dot{u}(t)=a u(t-\tau)-b u(t)-c u^{2}(t),
$$

where $a, b, c>0, u(t)>0$ for $-\tau \leq t \leq 0$, we have

(i) If $a>b$, then $\lim _{t \rightarrow+\infty} u(t)=\frac{a-b}{c}$.

(ii) If $a<b$, then $\lim _{t \rightarrow+\infty} u(t)=0$.

Now we state our permanence result for system (2).

Theorem 2.1. Let $M_{2}$ and $M_{3}$ be defined by (5), and (8), respectively. Suppose that the following conditions

$$
\alpha^{l} e^{-\gamma \tau}>a_{1}^{u} M_{3}+E^{u}, r_{1}^{l}>a_{2}^{u} M_{2}
$$

hold. Then system (2) is permanent, that is, there exist positive constants $m_{i}, M_{i}(i=1,2,3)$ which are independent of the solution of system $(2)$, such that for any positive solution $\left(x_{1}(t), x_{2}(t), y(t)\right)$ of system (2) with the initial condition $x_{i}(0)>0(i=1,2), y(0)>0$, one has

$$
\begin{gathered}
m_{i} \leq \lim _{t \rightarrow+\infty} \inf x_{i}(t) \leq \lim _{t \rightarrow+\infty} \sup x_{i}(t) \leq M_{i}, i=1,2 \\
m_{3} \leq \lim _{t \rightarrow+\infty} \inf y(t) \leq \lim _{t \rightarrow+\infty} \sup y(t) \leq M_{3}
\end{gathered}
$$

Proof. It is easy to see that system (2) with the initial value condition $\left(x_{1}(0)\right.$, $\left.x_{2}(0), y(0)\right)$ has positive solution $\left(x_{1}(t), x_{2}(t), y(t)\right)$ passing through $\left(x_{1}(0)\right.$, $\left.x_{2}(0), y(0)\right)$. Let $\left(x_{1}(t), x_{2}(t), y(t)\right)$ be any positive solution of system $(2)$ with the initial condition $\left(x_{1}(0), x_{2}(0), y(0)\right)$. It follows from the second equation of system (2) that

$$
\begin{aligned}
\frac{d x_{2}(t)}{d t} & =\alpha(t-\tau) e^{-\gamma \tau} x_{2}(t-\tau)-\beta(t) x_{2}^{2}(t)-a_{1}(t) x_{2}(t) y(t)-E(t) x_{2}(t) \\
& \leq \alpha(t-\tau) e^{-\gamma \tau} x_{2}(t-\tau)-\beta(t) x_{2}^{2}(t)-E(t) x_{2}(t) \\
& \leq \alpha^{u} e^{-\gamma \tau} x_{2}(t-\tau)-E^{l} x_{2}(t)-\beta^{l} x_{2}^{2}(t)
\end{aligned}
$$

It follows from (4) and Lemma 2.2 that

$$
\lim _{t \rightarrow+\infty} \sup x_{2}(t) \leq \frac{\alpha^{u} e^{-\gamma \tau}}{\beta^{l}}:=M_{2} .
$$

For any positive constant $\varepsilon>0$, it follows from (5) that there exists a $T_{1}>0$ such that for all $t>T_{1}$,

$$
x_{2}(t) \leq M_{2}+\varepsilon
$$


It follows from the third equation of system (2) that

$$
\begin{aligned}
\frac{d y(t)}{d t} & =y(t)\left(r_{1}(t)-a_{2}(t) x_{2}(t)-b(t) y(t)\right) \\
& \leq y(t)\left(r_{1}(t)-b(t) y(t)\right) \\
& \leq y(t)\left(r_{1}^{u}-b^{l} y(t)\right) .
\end{aligned}
$$

It follows from (7) and Lemma 2.1 that

$$
\lim _{t \rightarrow+\infty} \sup y(t) \leq \frac{r_{1}^{u}}{b^{l}}:=M_{3} .
$$

For any positive constant $\varepsilon>0$, it follows from (8) that there exists a $T_{2}>0$ such that for all $t>T_{2}$,

$$
y(t) \leq M_{3}+\varepsilon
$$

For any positive constant $\varepsilon>0$ and $T_{3}>T_{2}$, from the second equation of system (2), we have

$$
\begin{aligned}
\frac{d x_{2}(t)}{d t} & =\alpha(t-\tau) e^{-\gamma \tau} x_{2}(t-\tau)-\beta(t) x_{2}^{2}(t)-a_{1}(t) x_{2}(t) y(t)-E(t) x_{2}(t) \\
& \geq \alpha^{l} e^{-\gamma \tau} x_{2}(t-\tau)-\left[a_{1}^{u}\left(M_{3}+\varepsilon\right)+E^{u}\right] x_{2}(t)-\beta^{u} x_{2}^{2}(t) .
\end{aligned}
$$

Thus, as a direct corollary of Lemma 2.1, according to (10), one has

$$
\lim _{t \rightarrow+\infty} \inf x_{2}(t) \geq \frac{\alpha^{l} e^{-\gamma \tau}-\left[a_{1}^{u}\left(M_{3}+\varepsilon\right)+E^{u}\right]}{\beta^{u}} .
$$

Setting $\varepsilon \rightarrow 0$, it follows that

$$
\lim _{t \rightarrow+\infty} \inf x_{2}(t) \geq \frac{\alpha^{l} e^{-\gamma \tau}-\left(a_{1}^{u} M_{3}+E^{u}\right)}{\beta^{u}}:=m_{2} .
$$

For any positive constant $\varepsilon>0$ and $T_{4}>T_{1}$, from the third equation of system (2), we have

$$
\begin{aligned}
\frac{d y(t)}{d t} & =y(t)\left(r_{1}(t)-a_{2}(t) x_{2}(t)-b(t) y(t)\right) \\
& \geq y(t)\left(r_{1}^{l}-a_{2}^{u}\left(M_{2}+\varepsilon\right)-b^{u} y(t)\right) .
\end{aligned}
$$

Thus, as a direct corollary of Lemma 2.1, according to (13), one has

$$
\lim _{t \rightarrow+\infty} \inf y(t) \geq \frac{r_{1}^{l}-a_{2}^{u}\left(M_{2}+\varepsilon\right)}{b^{u}} .
$$

Setting $\varepsilon \rightarrow 0$, it follows that

$$
\lim _{t \rightarrow+\infty} \inf y(t) \geq \frac{r_{1}^{l}-a_{2}^{u} M_{2}}{b^{u}}:=m_{3} .
$$

Noting that the first equation of system (2) is equal to the following integration form

$$
x_{1}(t)=\int_{t-\tau}^{t} \alpha(s) e^{-\gamma(t-s)} x_{2}(s) d s .
$$


For any small positive constant $\varepsilon>0$, without loss of generality, we assume that $\varepsilon<\frac{1}{2} m_{2}$, it follows from (5) and (12) that there exists a $T_{5}>T_{3}$ such that

$$
m_{2}-\varepsilon<x_{2}(t)<M_{2}+\varepsilon \text { for all } t>T_{5} .
$$

Thus for $t>T_{5}+\tau$, it follows from (16) and (17) that

$$
x_{1}(t) \leq \int_{t-\tau}^{t} \alpha^{u}\left(M_{2}+\varepsilon\right) e^{-\gamma(t-s)} d s \leq \frac{3 \alpha^{u} M_{2}}{2 \gamma}\left(1-e^{-\gamma \tau}\right):=M_{1}
$$

and

$$
x_{1}(t) \geq \int_{t-\tau}^{t} \alpha^{l}\left(m_{2}-\varepsilon\right) e^{-\gamma(t-s)} d s \geq \frac{3 \alpha^{l} m_{2}}{2 \gamma}\left(1-e^{-\gamma \tau}\right):=m_{1} .
$$

Obviously, (5), (8), (12), (18) and (19) show that system (2) is permanent. The proof of Theorem 2.1 is complete.

\section{Example}

To illustrate the theoretical results, we consider the following example:

\section{Example 3.1.}

(20)

$$
\left\{\begin{aligned}
\frac{d x_{1}(t)}{d t}= & (60+\sin t) x_{2}(t)-\ln 3 x_{1}(t)-[60+\sin (t-1)] e^{-\ln 3} x_{2}(t-1), \\
\frac{d x_{2}(t)}{d t}= & {[60+\sin (t-1)] e^{-\ln 3} x_{2}(t-1)-(60+\cos t) x_{2}^{2}(t) } \\
& -(5-\cos t) x_{2}(t) y(t)-(4+\sin t) x_{2}(t), \\
\frac{d y(t)}{d t}= & y(t)\left(50+\cos t-(10+\cos t) x_{2}(t)-(40-\sin t) y(t)\right) .
\end{aligned}\right.
$$

Corresponding to system (2), one has $\alpha(t)=60+\sin t, \gamma=\ln 3, \tau=1$, $r_{1}(t)=50+\cos t, \beta(t)=60+\sin t, a_{1}(t)=5-\cos t, a_{2}(t)=10+\cos t$, $b(t)=40-\sin t, E(t)=4+\sin t$. It is easy to see that $\alpha^{u}=61, \alpha^{l}=59, a_{1}^{u}=6$, $a_{2}^{u}=11, r_{1}^{u}=51, r_{1}^{l}=49, \beta^{l}=59, b^{l}=39, E^{u}=5$. Then $M_{2}=0.3446$, $M_{3}=1.3077, \alpha^{l} e^{-\gamma \tau}=19.6667, a_{1}^{u} M_{3}+E^{u}=12.8462, a_{2}^{u} M_{2}=3.7906$. Then $\alpha^{l} e^{-\gamma \tau}>a_{1}^{u} M_{3}+E^{u}, r_{1}^{l}>a_{2}^{u} M_{2}$. Therefore all the conditions of Theorem 2.1 are satisfied which means that system (20) is permanent.

\section{Conclusions}

In this paper, we have investigated the dynamical behavior of a two species delayed competitive model with stage structure and harvesting. Sufficient conditions which ensure the permanence of the system are derived. It is shown that delay has influence on the permanence of system. Thus delay is an important factor to decide the permanence of the system. An example shows the feasibility of our main results. 


\section{References}

[1] J. R. Bence and R. M. Nisbet, Space-limited recruitment in open systems: the importance of time delays, Ecology 70 (1989), no. 5, 1434-1441.

[2] A. A. Berryman, The origns and evolution of predator-prey theory, Ecology 73 (1992), no. $5,1530-1535$.

[3] L. M. Cai and X. Y. Song, Permanence and stability of a predator-prey system with stage structure for predator, J. Comput. Appl. Math. 201 (2007), no. 2, 356-366.

[4] F. D. Chen, The permanence and global attractivity of Lotka-Volterra competition system with feedback control, Nonlinear Anal. Real World Appl. 7 (2006), no. 1, 133-143.

[5] - Permanence of a discrete $n$-species food-chain system with time delays, Appl. Math. Comput. 185 (2007), no. 1, 719-726.

[6] - Partial survival and extinction of a delayed predator-prey model with stage structure, Appl. Math. Comput. 219 (2012), no. 8, 4157-4162.

[7] F. D. Chen, W. L. Chen, Y. M. Wu, and Z. Z. Ma, Permanence of a stage-structured predator-prey system, Appl. Math. Comput. 219 (2013), no. 17, 8856-8862.

[8] F. D. Chen and M. S. You, Permanence for an integrodifferential model of mutualism, Appl. Math. Comput. 186 (2007), no. 1, 30-34.

[9] K. L. Cooke and P. van den Driessche, On zeroes of some transcendental equations, Funkcial. Ekvac. 29 (1986), no. 1, 77-90.

[10] J. M. Cushing, Periodic time-dependent predator-prey system, SIAM J. Appl. Math. 32 (1977), no. 1, 82-95.

[11] J. Dhar and K. S. Jatav, Mathematical analysis of a delayed stage-structured predatorprey model with impulsive diffusion between two predators territories, Ecol. Complex. in Press.

[12] Y. H. Fan and W. T. Li, Permanence for a delayed discrete ratio-dependent predatorprey model with Holling type functional response, J. Math. Anal. Appl. 299 (2004), no. $2,357-374$.

[13] Z. Y. Hou, On permanence of all subsystems of competitive Lotka-Volterra systems with delays, Nonlinear Anal. Real World Appl. 11 (2010), no. 5, 4285-4301.

[14] _ On permanence of Lotka-Volterra systems with delays and variable intrinsic growth rates, Nonlinear Anal. Real World Appl. 14 (2013), no. 2, 960-975.

[15] H. X. Hu, Z. D. Teng, and H. J. Jiang, On the permanence in non-autonomous LotkaVolterra competitive system with pure-delays and feedback controls, Nonlinear Anal. Real World Appl. 10 (2009), no. 3, 1803-1815.

[16] V. A. A. Jansen, R. M. Nisbet, and W. S. C Gurney, Generation cycles in stage structured populations, Bull. Math. Biol. 52 (1990), no. 3, 375-396.

[17] X. Y. Liao, S. F. Zhou, and Y. M. Chen, Permanence and global stability in a discrete $n$-species competition system with feedback controls, Nonlinear Anal. Real World Appl. 9 (2008), no. 4, 1661-1671.

[18] S. Q. Liu and L. S. Chen, Necessary-sufficient conditions for permanence and extinction in lotka-volterra system with distributed delay, Appl. Math. Lett. 16 (2003), no. 6, 911917.

[19] S. Q. Liu, L. S. Chen, and Z. J. Liu, Extinction and permanence in nonautonomous competitive system with stage structure, J. Math. Anal. Appl. 274 (2002), no. 2, 667684.

[20] X. Lv, S. P. Lu, and P. Lu, Existence and global attractivity of positive periodic solutions of Lotka-Volterra predator-prey systems with deviating arguments, Nonlinear Anal. Real World Appl. 11 (2010), no. 1, 574-583.

[21] Z. H. Ma, Z. Z. Li, S. F. Wang, T. Li, and F. P. Zhang, Permanence of a predator-prey system with stage structure and time delay, Appl. Math. Comput. 201 (2008), no. 1-2, $65-71$. 
[22] F. Montes de Oca and M. Vivas, Extinction in a two dimensional Lotka-Volterra sysstem with infinite delay, Nonlinear Anal. Real World Appl. 7 (2006), no. 5, 1042-1047.

[23] Y. Muroya, Permanence and global stability in a Lotka-Volterra predator-prey system with delays, Appl. Math. Lett. 16 (2003), no. 8, 1245-1250.

[24] R. M. Nisbet, W. S. C. Gurney, and J. A. J. Metz, Stage structure models applied in evolutionary ecology, in Applied Mathematical Ecology, Biomathematics, pp. 428-449, Springer, Berlin, Germany, 1989.

[25] X. Y. Song and L. S. Chen, Global asymptotic stability of a two species competitive system with stage structure and harvesting, Commun. Nonlinear Sci. Numer. Simul. 6 (2001), no. 2, 81-87.

[26] Z. D. Teng, Y. Zhang, and S. J. Gao, Permanence criteria for general delayed discrete nonautonomous-species Kolmogorov systems and its applications, Comput. Math. Appl. 59 (2010), no. 2, 812-828.

[27] J. Y. Wang, Q. S. Lu, and Z. S. Feng, A nonautonomous predator-prey system with stage structure and double time delays, J. Comput. Appl. Math. 230 (2009), no. 1, 283-299.

[28] R. Xu, L. S. Chen, and F. L. Hao, Periodic solutions of an n-species Lotka-Volterra type food-chain model with time delays, Appl. Math. Comput. 171 (2005), no. 1, 511-530.

[29] J. D. Zhao and J. F. Jiang, Average conditons for permanence and extinction in nonantonomous Lotka-Volterra system, J. Math. Anal. Appl. 299 (2004), no. 2, 663-675.

Changuin Xu

Guizhou Key Laboratory of Economics System Simulation

School of Mathematics and Statistics

Guizhou University of Finance and Economics

GUIYANG 550004, P. R. China

E-mail address: xcj403@126.com

YUSEN Zu

School of Mathematics and Statistics

Henan University of Science and Technology

Luoyang 471023, P. R. China

E-mail address: ylyyly2015@126.com 